\title{
Los consorcios, una nueva etapa de la cooperación bibliotecaria
}

\author{
Por Miquel Térmens Graells
}

\begin{abstract}
Resumen: El artículo analiza los consorcios de bibliotecas centrados en la adquisición de revistas electrónicas como una forma actualizada de cooperación bibliotecaria; muestra sus principios de actuación, basados en la teoría de juegos, así como los retos que su gestión interna presenta de cara a su permanencia como organizaciones.
\end{abstract}

Palabras clave: Consorcios bibliotecarios, Revistas electrónicas, Cooperación bibliotecaria.

\section{Title: The consortia: a new stage in library co- operation}

Abstract: This article analyses library consortia dedicated to electronic journal acquisition as a new kind of library co-operation. The text describes consortia behaviour rules, which are based on game theory, and in addition it shows how their internal management could represent a challenge for their survival as organisations.

Keywords: Library consortia, Electronic journals, Library co-operation.

Térmens Graells, Miquel. "Los consorcios, una nueva etapa de la cooperación bibliotecaria”. En: El profesional de la información, 2005, mayo-junio, v. 14, n. 3, pp. 166-173.

DIVERSOS AUTORES han analizado el papel de la cooperación entre las bibliotecas (Evans, 2002 , p. 275). Sus estudios indican que el término "cooperación" es justamente el que aparece con más frecuencia en la literatura profesional cuando se habla de proyectos conjuntos. Pero éste solamente es un primer nivel de interacción entre las instituciones participantes; le siguen, como etapas más avanzadas, la coordinación y la colaboración.

Si en el caso de la coordinación se pretende establecer acuerdos sobre la misión o los objetivos, en el campo de la colaboración se llegan a crear estructuras formales, incluso con carácter legal, y de trabajo en común (que incluyen alguna forma de autoridad) con la ejecución de actividades operativas y de planificación en base a un conjunto de misiones y objetivos acordados. Los consorcios bibliotecarios, una forma estable de cooperación con compromisos y objetivos a medio y largo plazo, pueden encajar en alguno de estos niveles y, con el tiempo y si tienen éxito, evolucionar hacia uno de naturaleza superior. Hay quien desde una perspectiva crítica ha añadido una nueva "c" entre
Miquel Térmens Graells es profesor de la Facultad de Biblioteconomía y Documentación de la Universidad de Barcelona, donde imparte asignaturas de edición electrónica, tecnología aplicada a las bibliotecas y políticas de información. Ha sido responsable de la Unidad técnica y de proyectos del Servicio de bibliotecas de la Universidad Autónoma de Barcelona.

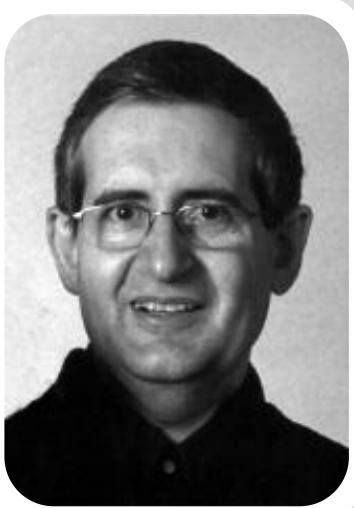

las posibles relaciones: la competición; las instituciones, incluidas las bibliotecas, compiten entre sí, sea para alcanzar la excelencia, para obtener recursos, para retener el favor de los usuarios o por otras razones (Peters, 2001b, p. 302; Peters, 2003a, p. 111).

La cooperación bibliotecaria existe desde finales del siglo XIX, como mínimo - es el caso de los EUA, donde se encuentra referenciada desde la década de 1880- Los consorcios como forma específica de cooperación también vienen de lejos, siendo Triangle Research Libraries Network, fundado en 1933, uno de los primeros (Bostick, 2001; Nfila, Darko-Amprem, 2002). Las bibliotecas cooperan porque obtienen beneficios en ello, como pueden ser el acceso a más documentación, soporte técnico, reducción de costes gracias al desarrollo de economías de escala, más visibilidad social, reforzamiento del poder de negociación ante los proveedores, acceso a nuevas fuentes de financiación, etc. Los intereses para crear y formar parte de un sistema de cooperación pueden ser, por tanto, varios; por esta razón en la mayoría de casos la cooperación se pone en marcha con unos fines concretos que 
después, si los resultados son positivos, se pueden ampliar.

Las décadas de 1960 y 1970 fueron de expansión de los consorcios bibliotecarios. La razón se encontraba en la necesidad de compartir recursos a nivel documental (mediante el préstamo interbibliotecario y otras fórmulas) y a nivel técnico (con la implantación de los primeros sistemas de catálogos automatizados). En algunos casos las asociaciones creadas evolucionaron hasta generar grandes redes de servicios compartidos, como fue el caso de $O C L C$ y de RLIN.

\section{«En algunos casos, las asociaciones creadas evolucionaron hasta generar grandes redes de servicios compartidos, como fue el caso de OCLC y de RLIN»}

Con la aparición de la información electrónica y su comercialización las prácticas de cooperación se han visto reforzadas. Las bibliotecas se asocian en forma de consorcios para negociar mejor los nuevos contratos, tener acceso a paquetes documentales que de forma individual no podrían, reclamar financiación especial para la transición hacia la documentación digital, y para darse soporte técnico mutuo. Como se puede observar, los beneficios obtenidos no son nuevos pero sí que lo es el objeto de la cooperación. El fenómeno se ha desarrollado sobre todo entre las bibliotecas académicas porque es en éstas donde la incorporación de la documentación digital se ha iniciado antes y donde lo está haciendo con una mayor importancia relativa. El proceso de asociación ha culminado con la aparición de un consorcio de consorcios: la International Coalition of Library Consortia (Icolc).

La comercialización de la información digital ha sido un factor clave en su desarrollo, pero también hay que recordar que este fenómeno se ha dado en un marco de reducción de la financiación que reciben las instituciones de educación superior y de una mayor presión para alcanzar servicios de calidad que aporten un mejor soporte a la investigación (Nfila, Darko-Ampem, 2002, p. 206). En este caso se trata de tendencias que presentan una incidencia muy diferente en cada país, pues mientras que en los EUA la reducción del presupuesto asignado a las bibliotecas es evidente, en España, afortunadamente, más bien se ha dado el proceso inverso, eso sí, partiendo de unos niveles muy bajos (Kohl, 2003, p. 53).

En el contexto europeo la expansión de los consorcios también es un fenómeno reciente, de los años 90. Su estudio muestra que se ha extendido por la ma- yoría de países pero con características diversas en cada uno de ellos, con instituciones de cobertura estatal (Francia, Reino Unido, etc.) y otras de carácter regional (Alemania, España, etc.) (Giordano, 2000). En una reciente encuesta, la comparación entre la fecha de fundación de importantes consorcios europeos y norteamericanos mostraba cómo los primeros son mucho más jóvenes que los segundos, tal como indica el dato de que, entre los europeos, sólo el 17\% apareció antes del año 1990 mientras que así lo hicieron el 60\% de los norteamericanos (King; Xu, 2002, p. 38).

\section{Consorcios centrados en la adquisición de revistas-e}

Actualmente uno de los principales objetivos perseguidos es la adquisición conjunta de información digital, hasta el punto de que ésta es la finalidad única y la razón de ser de algunas de estas instituciones. Para algunos autores se han convertido en clubs de compra (buying clubs) (Bostick, 2001, p. 129). Según Tommaso Giordano (2002) se debe principalmente a una falta de explicitación de su misión como institución, en tanto que los objetivos y los programas de actuación sí que lo están; con todo, muchos consorcios no van más allá de una mera compra de licencias. Para Tom Sanville, director ejecutivo de OhioLINK, posiblemente el consorcio más avanzado e influyente del mundo, la visión es bien distinta:

"Podemos vencer las limitaciones inherentes al medio impreso, las prácticas económicas atrincheradas y restrictivas de los vendedores a las instituciones individuales, así como la extendida mentalidad limitadora de racionar la información que se autoimpone la propia comunidad bibliotecaria. Cambiando radicalmente la ecuación de la información suministrada por cada dólar gastado, a largo plazo los consorcios pueden situar la evolución de nuestra industria a una nueva y mejor posición" (Sanville, 1999, p. 47).

Los consorcios no son, pues, simplemente un instrumento para adquirir información bajo una nueva forma, sino una herramienta de transformación de las prácticas bibliotecarias hacia un modelo de gestión y de servicio aún en fase de definición.

Aunque la adquisición consorciada se puede aplicar a cualquier tipo de información ${ }^{1}$ y a otros productos como el software (Peters, 2002b, p. 248), la forma más extendida y con un mayor impacto tanto económico como de servicios al usuario es la contratación de revistas electrónicas.

La negociación de los contratos de adquisición de información digital está considerada como difícil, ya sea a nivel técnico o financiero (Harris, 2001). Hacerlo de forma conjunta dentro de un consorcio es una es- 
trategia para minimizar los riesgos de una mala negociación y, a la vez, tener más capacidad negociadora ante los proveedores. La mayoría de las partes que intervienen en una operación comercial consideran que obtienen un provecho si alcanzan un beneficio económico que, en el caso de los compradores, se concreta en una rebaja del precio. Algunos autores advierten que no siempre es así, pues un buen negociador puede obtener beneficios de otro tipo y éste es un fenómeno que han de tener presente las instituciones que se incorporan a un sistema de negociación consorciada (Reader, 1999; King, Xu, 2002, pp. 15-17). También se indica que al pasar de un sistema de precios fijos (las tarifas más o menos públicas de las suscripciones individuales) a otro con negociación (los contratos por paquetes) las instituciones pierden buena parte de las referencias comparadas que hasta entonces habían utilizado como guía para su toma de decisiones: cada contrato ha de ser "negociado según su propio valor, en el contexto de lo que ese producto, a un precio determinado, con unos términos concretos de la licencia significa para una comunidad específica [...]; cada situación de licencia es única" (Miller, 1999, p. 113).

\section{«Actualmente uno de los principales objetivos a conseguir es la adquisición conjunta de información digital, hasta el punto de que esta es la finalidad única y la razón de ser de algunas de estas instituciones»}

Más allá de las virtudes de la cooperación, hay quien opina que el objetivo final de la mayoría de instituciones es sobrevivir ${ }^{2}$ y que la utilización de los consorcios para alcanzarlo se produjo durante los 90: "el desarrollo más importante de las bibliotecas académicas durante la década actual ha sido el cambio de modelo desde la auto-suficiencia institucional a un modo de sobrevivir colaborativo, plasmado en el crecimiento de los consorcios bibliotecarios" (Allen; Hirshon, 1998, p. 36). Si así es, los consorcios centrados en la adquisición de revistas electrónicas son básicamente mecanismos defensivos ante los editores y los problemas financieros que se han de soportar. Para otros (Piotrowicz, 2002) son instrumentos más amplios de colaboración entre bibliotecas, que pueden evolucionar a lo largo del tiempo. En este sentido se han identificado distintas actividades para la acción futura:

- conformar nuevos sistemas de precios para la distribución de información electrónica;

—negociar acuerdos de licencias; —facilitar la implementación de nuevas tecnologías;

- coordinar proyectos de uso compartido de recursos;

- promover la mejora de la infraestructura de información, y

—resolver problemas de gestión y de financiación.

Desde el punto de vista teórico, la adquisición consorciada de revistas electrónicas ha sido analizada como un caso típico de aplicación de la teoría de juegos: los consorcios son una forma de negociación "social" para obtener un bien para todos los socios por encima de los intereses egoístas de tipo personal; de aquí la repetida frase de sus impulsores: "todo el mundo gana con los consorcios" (Hayes, 2003). El famoso dilema del prisionero ahora se ha convertido en el del bibliotecario: si colaboro me salvo a costa de diluir mi identidad en la de las otras bibliotecas, pero si no lo hago puedo alcanzar la excelencia con una solución apropiada a mis características... o quizá no tendré éxito en el mundo digital ${ }^{3}$. Utilizando otra teoría económica, la tragedia de las tierras comunales ${ }^{4}$, Philip M. Davis argumenta que los casos del sistema de publicación científica y el del papel de la biblioteca de investigación son similares: más allá de la cooperación (posiblemente un mecanismo que sólo les ata aún más con los intereses de los editores) los estudiantes y el personal docente e investigador utilizan la biblioteca como un bien público, de forma egoísta y sin ninguna responsabilidad respecto a su agotamiento como recurso común. Desde esta actitud presionan con la finalidad de que la biblioteca asuma aquello que antes eran subscripciones de carácter individual, dando como resultado un beneficio particular para los antiguos subscriptores pero a costa de un mal común, como es el aumento del gasto destinado a las subscripciones institucionales (Davis, 2003). En la misma línea, Bergstrom y Bergstrom también han analizado los intereses enfrentados de los editores, de las bibliotecas y de los lectores académicos para concluir que, sin una colaboración interbibliotecaria, las decisiones institucionales individuales tenderán a reforzar el sistema de licencias por paquetes frente a las subscripciones individuales, y con este cambio favorecerán la maximización de los beneficios de los editores (Bergstrom; Bergstrom, 2004). Una tesis doctoral sobre el caso del Consorci de Biblioteques Universitàries de Catalunya (CBUC) también analiza al amparo de la teoría de juegos los beneficios que la cooperación reporta a las bibliotecas asociadas, concluyendo que todas salen beneficiadas de una forma u otra (Sales, 2002).

Si los beneficios económicos son los más evidentes y fáciles de cuantificar, no se puede olvidar que también se pueden conseguir ventajas de otro tipo. Al- 
gunos análisis de coste-beneficio también empiezan a estudiar los ahorros en el tiempo que obtienen el personal académico y los estudiantes por el hecho de evitarse desplazamientos y disponer de una oferta más amplia de revistas; este tiempo ahorrado tiene, naturalmente, una equivalencia monetaria que se puede cuantificar (Scigliano, 2002, p. 397).

Con independencia de cuáles puedan ser sus efectos comprobados, la realidad es que el sistema de consorcios se ha generalizado: cada vez existen más y algunos de éstos se llegan a federar en "meta-consorcios". En los países donde este fenómeno se encuentra más desarrollado, como en EUA, no es raro que una misma biblioteca forme parte de varios consorcios de los que obtiene servicios diferentes. Algunos autores alertan sobre la competición encubierta que se establece para captar nuevos miembros o para ofrecerles mejores prestaciones que la competencia. Esta multiafiliación puede atar a las bibliotecas aún más a este modelo y también afectar a los editores, que se encuentran con una clientela cada vez más comprometida por acuerdos externos y con menos capacidad de acción individual (Peters, 2001a).

Otra modalidad interesante es la de los acuerdos de base estatal. La disponibilidad de los grandes proveedores a negociar contratos de amplia base, así como la necesidad de que éstos dispongan de algún tipo de financiación adicional por parte del gobierno respectivo, ha llevado en algunos casos a la creación de consorcios de alcance estatal para proveer determinados contenidos a todas las instituciones de educación superior y de investigación de un país, o incluso a toda la población de un territorio. El ejemplo más exitoso de la primera opción es el consorcio británico Nesli (Turner, 1999; Woodward, 2000), mientras que en la segunda cabe destacar el caso de Islandia (Hallgrimsson, 2004).

\section{Los consorcios en el mundo}

La expansión mundial de los consorcios centrados en la adquisición de recursos electrónicos y la coincidencia de los problemas y de las necesidades en la mayoría de los mismos llevó a la constitución de una asociación que los representase, la International Coalition of Library Consortia (Icolc) $)^{5}$. Nació en el año 1996 bajo la denominación de Consortium of Consortia $(C O C)$, adoptando en el siguiente año el actual nombre. Desde entonces funciona como una organización informal que principalmente actúa como medio de consulta entre las instituciones asociadas y como portavoz común para la defensa de sus intereses. Su infraestructura es mantenida por la Yale University Library y se reduce a un sitio web y a una lista de distribución de acceso restringido a sus miembros.
Los miembros de la Icolc, actualmente unas 150 instituciones, se reúnen en dos congresos anuales en los EUA (desde el año 1997) y en uno anual del grupo europeo (desde 1999). A lo largo de estos años su aportación más tangible han sido los documentos que presentaban un posicionamiento colectivo respecto a cuestiones concretas de su relación con los proveedores. Así, destacan las pautas para la medida estadística del uso de los recursos electrónicos (Icolc, 1998) y las recomendaciones de buenas prácticas para la selección y adquisición de información electrónica (Icolc, 2001; Foster, 2003; Tonkery, 2003). Ambos documentos han tenido una gran repercusión e influencia: por un lado demostraban que desde las bibliotecas se podían presentar a los proveedores propuestas sólidas a nivel técnico y jurídico que defendieran sus intereses. Además, la Icolc también obtenía el reconocimiento de interlocutor válido por parte de los grandes editores, con lo que se alcanzaba uno de los objetivos primarios del movimiento de consorcios de convertirse en un contrincante fuerte para plantar cara a los vendedores. Alcanzados estos hitos, la coalición parece haber perdido últimamente una parte de su antigua actividad, como si después de haber sido reconocido su status de lobby de poder ante los editores, los intereses y las estrategias diversas entre los consorcios representados impidieran una actuación común más reforzada.

\section{«Muchos consorcios inicialmente centrados en la adquisición de documentación digital han ampliado sus actividades hacia otras formas de cooperación»}

Las dificultades para articular un posicionamiento común entre las bibliotecas o entre los distintos consorcios también pueden haber afectado negativamente en el intento fracasado de dotarse de una revista especializada en la materia: Library consortium management: an international journal (ISSN 1466-2760). Publicada por MCB University Press, su primer número apareció en 1999. Inicialmente deseaba convertirse en una plataforma pública de discusión entre los diversos sectores implicados (editores, bibliotecas y agentes de subscripciones) y también actuar como caja de resonancia y de difusión de las actividades de los diversos consorcios. Bien pronto se constató que no existían suficientes originales para llenar sus páginas y los nuevos números pronto redujeron su extensión, dejando de publicarse el siguiente año después de una historia de 8 volúmenes.

En España, la aparición de los consorcios es un fenómeno reciente respecto a otros países. No es más 
que una de las consecuencias del retraso en su desarrollo bibliotecario, en especial en la creación de estructuras de gestión profesional potentes, el inicio en la automatización de los catálogos y la introducción de la informática dentro de los servicios, así como la disponibilidad de presupuestos suficientes y de márgenes de confianza para su aplicación en una época de cambios (Anglada, 1995; Anglada, 2003; Merlo, 1999).

Si la cooperación en general ha sido baja, aquella centrada en la adquisición de materiales aún ha sido menor y quizás se pueda considerar como inexistente, si dejamos de lado las colaboraciones en el préstamo interbibliotecario, hasta la aparición de la edición electrónica. El primer consorcio creado en España con este nombre, en línea con las tendencias internacionales, con un régimen jurídico formal, con una estructura y una financiación propias, y siguiendo una dirección clara hacia unos objetivos a medio y largo plazo, fue el Consorcio de Bibliotecas Universitarias de Cataluña $(C B U C)$, fundado en 1995. De forma significativa, el objetivo inicial fue la creación de un catálogo colectivo y la obtención de ahorros gracias a la catalogación compartida. Unos años más adelante, con la puesta en marcha del servicio llamado Biblioteca Digital de Catalunya, entró en la adquisición conjunta de documentación electrónica: revistas, libros y bases de datos. Su ejemplo pionero, así como la influencia del movimiento internacional, ha facilitado que posteriormente se hayan establecido otros:

\section{—Fundació Biblioteca Josep Laporte.}

- Consorcio de Universidades de la Comunidad de Madrid y de la UNED para la Cooperación Bibliotecaria (Madroño).

- Consorcio de Bibliotecas Universitarias de Andalucía (CBUA).

-Consorcio de Bibliotecas Universitarias de Galicia (Bugalicia).

\section{La gestión de los consorcios}

Tal como indica la evolución de algunas de estas instituciones, muchos consorcios inicialmente centrados en la adquisición de documentación digital han ampliado sus actividades hacia otras formas de cooperación, muchas veces sin abandonar el nuevo ámbito digital. Son un ejemplo de estas tendencias aquellos creados para:

-mantener repositorios colectivos de documentación digital (Peters, 2002a; Prosser, 2003);

- ofrecer servicios de referencia electrónica (Peters, 2002b), o
- dar soporte a la educación a distancia (Subramanian, 2002).

La ampliación de sus actividades también provoca el aumento de la complejidad de su organización interna y la necesidad de buscar mayores compromisos que hagan posible alcanzar sus objetivos. Ésta es la razón por la que muchas colaboraciones de carácter informal evolucionan con el paso del tiempo hacia compromisos formalizados y reglamentados (Wade, 1999). Ahora bien, incluso en los casos centrados exclusivamente en la adquisición de documentación digital, la cooperación bajo la forma jurídica de consorcio no es garantía de éxito. No se trata de valorar si su forma de abordar la adquisición de revistas electrónicas es más o menos favorable para las bibliotecas, sino si la propia estructura de consorcio como tal presenta puntos débiles. Chan y Ferguson (Chan; Ferguson, 2002, pp. 16-19) relacionan un total de siete retos que se han de superar si un consorcio bibliotecario desea alcanzar el éxito:

- La colaboración requiere tiempo y energía. Es una actividad "no natural" y las instituciones se resisten a comprometer de forma voluntaria sus intereses individuales. El resultado es un gran consumo de tiempo y de energías para desarrollar todas las acciones administrativas que van ligadas a un proceso de negociación, identificación de las necesidades, organización de pruebas de productos, evaluación de éstos, negociación con los vendedores, cálculo del impacto económico del gasto, determinación del proceso de pago, distribución de los costes entre los miembros del consorcio, así como el control y monitorización del sistema de acceso. Una vez se ha firmado el contrato, el trabajo aún no se acaba pues se han de obtener e interpretar las estadísticas de uso y comunicar al proveedor los problemas que en la prestación del servicio observa cada uno de los miembros consorciados.

- Insuficiente poder de compra. Los consorcios desean obtener ahorros económicos por unidad, hecho que va en contra de los intereses del proveedor; a éste sólo lo pueden convencer ofreciéndole un mayor volumen global de ventas gracias a la incorporación de más clientes. De aquí se deduce la presión interna de los consorcios para crecer pues ello les hace ganar poder negociador.

- Superposición de la afiliación a diversos consorcios. Las presiones para crecer llevan a una competencia entre ellos para obtener nuevos afiliados. Las tensiones que este fenómeno origina pueden provocar que algunos consorcios casi actúen como representantes o distribuidores de determinados grupos editoriales, rompiendo con su naturaleza fundacional. 
-Falta de personal directivo con las aptitudes necesarias para negociar contratos. La agregación de los talentos de diversas instituciones no significa que entre ellos se encuentren las habilidades para concertar correctamente los contratos.

-Diferencias en los beneficios obtenidos por determinadas bibliotecas. Algunos centros obtenían productos con un menor precio con contratos individuales que después formando parte de un consorcio; puede ser debido a que formaban parte de grupos promocionales o a compensaciones recibidas por otras relaciones comerciales. Otro motivo de diferencias, éste mucho más frecuente, es un incremento diferente en el número de títulos obtenidos gracias a un contrato consorciado: cuando en un consorcio el número de subscripciones previas varía mucho entre las diversas instituciones, las que tenían un número menor ganan más títulos gracias al consorcio que las que ya disponían de una buena colección. En este caso, las comparaciones por el incremento del valor obtenido en relación con el gasto consolidado aportado pueden ser muy duras.

\section{«Los consorcios amplían sus áreas de actuación y también intentan crecer en volumen porque ello les otorga una mayor capacidad de negociación ante los proveedores»}

-Diferencias entre las necesidades y los intereses. No todos los productos que se ofrecen representan el mismo valor para cada institución, y mientras unas pueden ser muy favorables a un aumento del volumen de documentación disponible a cambio de un bajo incremento en el coste, para otras es inaceptable pues rompe con su concepto de colección.

-Diferencias en el volumen y nivel de flexibilidad de los presupuestos. Este problema se puede presentar bajo múltiples formas: presupuestos insuficientes, ya comprometidos en otras líneas de acción, no disponibilidad de presupuesto en el momento que se firman los contratos o fragmentación de los fondos en partidas de destinación temática que impiden participar en la adquisición de productos de composición generalista.

Los consorcios amplían sus áreas de actuación y también intentan crecer en volumen porque esto les otorga una mayor capacidad de negociación ante los proveedores. El resultado en muchos casos es un incremento en la complejidad del sistema de afiliación: a los miembros de pleno derecho de un consorcio se les suman otras instituciones afiliadas, con niveles va- riables de compromiso, que normalmente sólo participan de forma parcial en los objetivos consorciados o que sólo disfrutan de un servicio o contrato determinado (Peters, 2003b).

Los contratos consorciados están de moda, lo que puede inducir a algunas instituciones a adherirse a uno de ellos sin medir de forma suficiente los resultados que obtendrán. Stern (2003, p. 156) recomienda examinar tres aspectos clave para tomar la decisión de forma adecuada:

- En un servicio o producto determinado ¿son superiores los costes ocultos de la infraestructura consorciada a los ahorros y beneficios alcanzados con el contrato conjunto?

— ¿La motivación para integrarse en un consorcio reside en el interés hacia un producto o existen otros motivos como, por ejemplo, querer participar en decisiones estratégicas?

— ¿Qué es más importante para la institución: disponer de un buen control de los costes y capacidad de decisión sobre la selección y modificación de títulos, o bien obtener costes reducidos a costa de un control limitado?

También, con el objetivo de racionalizar los resultados obtenidos gracias a los contratos por paquetes, se ha propuesto que las instituciones apliquen técnicas de benchmarking con las que medir a posteriori y de forma objetiva el balance de estos servicios, como ya se aplican en el Southern Universities Purchasing Consortium (SUPC) del Reino Unido (Wright, 2003) o en el consorcio norteamericano OhioLINK (Gatten, 2004).

Desde la óptica de la propia organización del consorcio surge otro problema con el paso del tiempo: la redistribución de los costes operativos. Con el aumento de las actividades y la firma de nuevos contratos no sólo varía el volumen del presupuesto gestionado sino también el grado de participación y de beneficios que obtienen los diversos miembros; este fenómeno se agrava en caso de que el consorcio crezca con nuevos afiliados. Con estas variables, el establecimiento de baremos transparentes y lo más objetivo posibles para la redistribución de las cargas se convierte en una de las preocupaciones centrales de los equipos directivos (Sloan, 1998; Sloan, 1999).

\section{Cooperación y sistema bibliotecario}

La cooperación bibliotecaria por medio de consorcios se ha convertido en una actividad robusta; así lo indica el gran número de los existentes y sus evidentes logros al conseguir un aumento de las colecciones disponibles para los usuarios. También, como hemos ob- 
servado, es un movimiento que se pregunta por su continuidad y por su evolución como institución viva.

\section{«Los contratos consorciados están de moda, lo que puede inducir a algunas instituciones a adherirse a uno de ellos sin medir de forma suficiente los resultados que obtendrán»}

Hay que resaltar que, más allá de sus propósitos iniciales para reformar el sistema de adquisiciones de revistas electrónicas, se están convirtiendo en instrumentos para transformar directa o indirectamente el actual sistema bibliotecario, en especial en el mundo académico. Para algunos de sus teóricos más reputados, las bibliotecas deben cambiar de forma radical no sólo sus sistemas de adquisiciones sino también las bases sobre las que se asienta la selección de la colección documental que deben o pueden ofrecer a sus lectores (Kohl, 2003). Para ello, los consorcios han de demostrar que además de ser instrumentos válidos para contratar documentación por lotes son capaces de aportar soluciones para gestionarlas adecuando la oferta a su uso real, sin que por ello las particularidades de las distintas bibliotecas consorciadas se diluyan dentro de los intereses homogeneizadores del consorcio (Gatten; Sanville, 2004)

\section{Notas}

1. La adquisición de libros electrónicos es un ámbito emergente (un ejemplo en: Langston, 2003), que también presenta diferencias con el de las revistas electrónicas en lo que respecta a los posibles modelos de contratación (véase el estudio exploratorio de Hardy, Oppenheim, Rubbert, 2001).

2. Esta es la opinión de Ross Atkinson, de la Cornell University (Atkinson, 2001, p. 6), citado por Peters (2001b, p. 302). También Osorio, 2002, p. 2.

3. Frazier, 2001. Una respuesta directa en To the editor, 2001.

4. Los pastos comunales de un pueblo son un bien común; cada granjero lleva sus vacas a pastar allí, de lo que saca un beneficio particular; su tentación es llevar una nueva vaca, sabe que esto supondrá una redistribución de los pastos disponibles entre un mayor número de animales, con lo que cada uno recibirá menos, pero para este granjero la pérdida será pequeña en comparación con el beneficio de disponer de una nueva vaca pastando. En resumen: los granjeros individuales tienden a maximizar sus beneficios individuales aunque ello sea en contra del beneficio global de todos los granjeros. En una situación extrema, el egoísmo individual puede llevar al colapso de todo el sistema porque habrá tantas vacas en el sistema que no se dispondrá de alimento para todas y el recurso, el pasto, se agotará.

\section{5. http://www.library.yale.edu/consortia/}

\section{Referencias bibliográficas}

Allen, Barbara McFadden; Hirshon, Arnold. «Hanging together to avoid hanging separately: opportunities for libraries and consortia». En: Information technology in libraries, 1998, v. 17, n. 1, pp. 36-44.

Anglada, Lluís. «Cooperació bibliotecària a Espanya». En: Item, 1995, n. 16, pp. 51-67.
Anglada, Lluís. Cooperación entre bibliotecas universitarias españolas y la experiencia del CBUC. Consultado en: 20-12-04.

http://internet.cervantes.es/internetcentros/biblioteca/Coobiblioestambul/ ponencia_esp_llan.htm

Atkinson, Ross. «Contingency and contradiction: the place(s) of the library at the dawn of the new millennium». En: Journal of the American Society for Information Science and Technology, 2001, v. 52, n. 1, pp. 3 11.

Bergstrom, Carl T.; Bergstrom, Theodore C. "The costs and benefits of library site licenses to academic journals". En: PNAS, 2004, v. 101, n. 3 , pp. 897-902.

Bostick, Sharon L. "The history and development of academic library consortia in the United States: an overview". En: The journal of academic librarianship, 2001, v. 27, n. 2, pp. 128-130.

Chan, Gayle; Ferguson, Anthony W. «Digital library consortia in the 21st century: the Hong Kong Julac case». En: Collection management, 2002, v. 27 , n. $3 / 4$.

Davis, Philip M. "Tragedy of the commons revisited: librarians, publishers, faculty and demise of a public resource". En: Portal, 2003, v. 3, n. 4, pp. 547-562.

Evans, G. Edward. «Management issues of consortia. Part two». En: Library management, 2002 , v. 23 , n. 6/7, pp. 275-286.

Foster, Connie, [et al.]. «International Coalition of Library Consortia Statement of current perspective and preferred practices for the selection and purchase of electronic information: update n. 1 and reactions from the scholarly community». En: Serials review, 2003, v. 29, n. 1, pp. 3-10.

Frazier, Kenneth. «The librarians' dilemma. Contemplating the costs of the 'big deal'». En: D-Lib magazine, 2001, v. 7, n. 3. Consultado en: 2012-04.

http://www.dlib.org/dlib/march01/frazier/03frazier.html

Gatten, Jeffrey N. "Measuring consortium impact on user perceptions: OhioLINK and LibQUAL+". En: The journal of academic librarianship, 2004, v. 30, n. 3, pp. 222-228.

Gatten, Jeffrey N.; Sanville, Tom. "An orderly retreat from the big deal. Is it possible for consortia?”. En: D-Lib magazine, 2004, v. 10, n. 18.

Giordano, Tommaso. "Digital resource sharing and library consortia in Italy". En: Information technology and libraries, 2000, v. 19, n. 2, pp. 8489

Giordano, Tommaso. «Library consortium models in Europe: a comparative analysis". En: Alexandria, 2002, v. 14, n. 1, pp. 41-52.

Hallgrimsson, Thorsteinn. "Consortium finance: the way ahead or a dead end?". En: ASA Conference, 2004. Consultado en: 20-12-04.

http://www.subscription-

agents.org/conference/200402/thorsteinn.hallgimsson.pps

Hardy, Rachel; Oppenheim, Charles; Rubbert, Iris. "Pricing strategies and models for the provision of digitized text in higher education". En: Journal of information science, 2002, v. 28, n. 2, pp. 97-110.

Harris, Lesley Ellen. "How to be a better negotiator in the digital realm”. En: Learned publishing, 2001, v. 14, n. 4, pp. 303-306.

Hayes, Robert M. «Cooperative game theoretic models for decisionmaking in contexts of library cooperation». En: Library trends, 2003, v. 51, n. 3, pp. 441-461.

International Coalition of Library Consortia (Icolc): Guidelines for statistical measures of usage of web-based indexed, abstracted, and full-text resources. Consultado en: 20-12-04.

http://www.library.yale.edu/consortia/webstats.html

International Coalition of Library Consortia (Icolc): Statement of current perspective and preferred practices for the selection and purchase of electronic information: update n. 1: new developments in e-journal licensing (December 2001 update to March 1998 statement). Consultado en: 20-12-04.

http://www.library.yale.edu/consortia/2001currentpractices.htm 


\title{
Versión online de EPI
}

Existe una versión electrónica de El profesional de la información, de uso gratuito para todos los suscriptores de la revista, que pueden acceder a través de internet a los textos completos y materiales gráficos publicados desde el año 2000.

\author{
Más información en: \\ http://www.elprofesionaldelainformacion.com/contenidos.html
}

King, Donald W.; Xu, Hong. "The role of library consortia in electronic journal services". En: The consortium site licence: is it a sustainable model? Oxford: Ingenta Institute, 2002. pp. 9-76. Isbn 0-9541867-1-0.

Langston, Marc. «The California State University E-book Pilot Project: implications for cooperative collection development». En: Library collections, acquisitions \& technical services, 2003, v. 7, pp. 19-32.

Kohl, David. "Consortial licensing vs. tradition: breaking up is hard to do". En: Learned publishing, 2003, v. 16, n. 1, pp. 47-53.

Merlo Vega, José Antonio. "La cooperación en las bibliotecas universitarias: fundamentos y redes cooperativas". En: Boletín de la Asociación Andaluza de Bibliotecarios, 1999, n. 54, pp. 33-57.

Miller, Lindsey, [et al.]. «A research review for librarians working with electronic serials and licensing agreements in the age of the internet and distance education». En: The bottom line: managing library finances, 1999, v. 12, n. 3, pp. 113-119.

Nfila, Reason Baathuli; Darko-Ampem, Kwasi. «Developments in academic library consortia from the 1960s through to 2000: a review of the literature». En: Library management, 2002, v. 23, n. 4/5, pp. 203-212.

Osorio, Nestor L. «Consortia and the big deals: a new way of doing business or the end of the technical library?». En: Iatul proceedings, 2002. Consultado en: 20-12-04.

http://www.iatul.org/conference/proceedings/vol12/papers/osorio.pdf

Peters, Thomas A. "Overlap and competition among consortia for e-resource agreements". En: The Charleston advisor, 2001, v. 2, n. 3.

Peters, Thomas A. «What's the big deal?». En: The journal of academic librarianship, 2001, v. 27, n. 4, p. 302-304.

Peters, Thomas A. «Digital repositories: individual, discipline-based, institutional, consortial, or national?». En: The journal of academic librarianship, 2002, v. 28, n. 6, pp. 414-417.

Peters, Thomas A. «E-reference: how consortia add value». En: The journal of academic librarianship, 2002, v. 28, n. 4, pp. 248-250.

Peters, Thomas A. «Consortia and their discontents». En: The journal of academic librarianship, 2003, v. 29, n. 2, pp. 111-114.

Peters, Thomas A. «Graduated consortial memberships and rogue facilitators». En: The journal of academic librarianship, 2003, v. 29, n. 4, pp. 254-256.

Piotrowicz, Grayna. «The university libraries consortia: yesterday, today and tomorrow». En: EBIB: electronic information bulletin for librarians, 2002, n. 7. Consultado en: 20-12-04.

http://ebib.oss.wroc.pl/english/grant/piotrowicz.php

Prosser, David C. «The next information revolution: can institutional repositories and self-archiving transform scholarly communications?». En: Iatul proceedings, 2003. Consultado en: 20-12-04. http://www.iatul.org/conference/proceedings/vol13/papers/PROSSER_fullt ext.pdf

Reader, Evan A. "Purchasing reosurces collectively: what makes a consortium successful?". En: The Charleston advisor, 1999, v. 1, n. 2.
Sales Zaguirre, Jordi. Models cooperatius d'assignació de costos en un consorci de biblioteques. [Barcelona], Universitat de Barcelona. Tesis doctoral del Departamento de Matemática Económica, Financiera y Actuarial, 2002. Isbn 84-688-1742-2. Consultado en: 20-12-04. http://www.tdx.cesca.es/TDCat-0318103-161333/

Sanville, Tom. «Use levels and new models for consortial purchasing of electronic journals». En: Library consortium management, 1999, v. 1, n. 3/4, pp. 47-58.

Scigliano, Marisa. «Consortium purchases: case study for a cost-benefit analysis». En: The journal of academic librarianship, 2002, v. 28, n. 6, pp. 393-399.

Sloan, Bernie. «Allocating costs in a consortial environment: a methodology for library consortia». En: The bottom line: managing library finances, 1998, v. 11, n. 2, pp. 65-71.

Sloan, Bernie. «Allocating costs in a consortial environment: a methodology for library consortia». En: OCLC systems \& services, 1999, v. 15, n. 1 , pp. $45-52$.

Stern, David. "Comparing consortial and differential pricing models". En: The bottom line: managing library finances, 2003, v. 16, n. 4, pp. 154-156.

Subramanian, Jane M. «The growing and changing role of consortia in providing direct and indirect support for distance higher education». En: The reference librarian, 2002, n. 77, pp. 39-62.

"To the editor [letters received in response to the opinion piece, 'The librarians' dilemma: contemplating the costs of the «big deal»', by Kenneth Frazier in the March 2001 issue of D-lib magazine]". En: D-lib magazine, 2001, v. 7, n. 4. Consultado en: 20-12-04. http://www.dlib.org/dlib/april01/04letters.html

Tonkery, Dan. «A middleman's view to Icolc's 'update n. 1'». En: Serials review, 2003, v. 29, n. 1, pp. 6-8.

Turner, Rollo. «National site licensing and consortia purchasing». En: $L i$ brary consortium management, 1999, v. 1, n. 1/2, pp. 33-40.

Wade, Rona. «The very model of a modern library consortium». En: $L i$ brary consortium management, 1999, v. 1, n. 1/2, pp. 5-18.

Wright, Susan. "Benchmarking of deals". En: E-Icolc (International Coalition of Library Consortia in Europe) Conference, 2003. Consultado en: 21-12-04

http://www.deflink.dk/upload/doc_filer/doc_alle/1295_Benchmarking deals E-ICOLC.ppt

Woodward, Hazel. «Licensing e-journals: UK style». En: The serials librarian, 2000, v. 42, n. 1/2, pp. 135-141.

Miquel Térmens Graells, profesor de la Facultad de Biblioteconomía y Documentación de la Universidad de Barcelona.

termens@ub.edu 